\title{
Indicadores
}

\section{Clasificación integrada de revistas científicas (CIRC): propuesta de categorización de las revistas en ciencias sociales y humanas}

\author{
Por Daniel Torres-Salinas, María Bordons, Elea Giménez-Toledo, Emilio Delgado-López-Cózar, \\ Evaristo Jiménez-Contreras y Elías Sanz-Casado
}

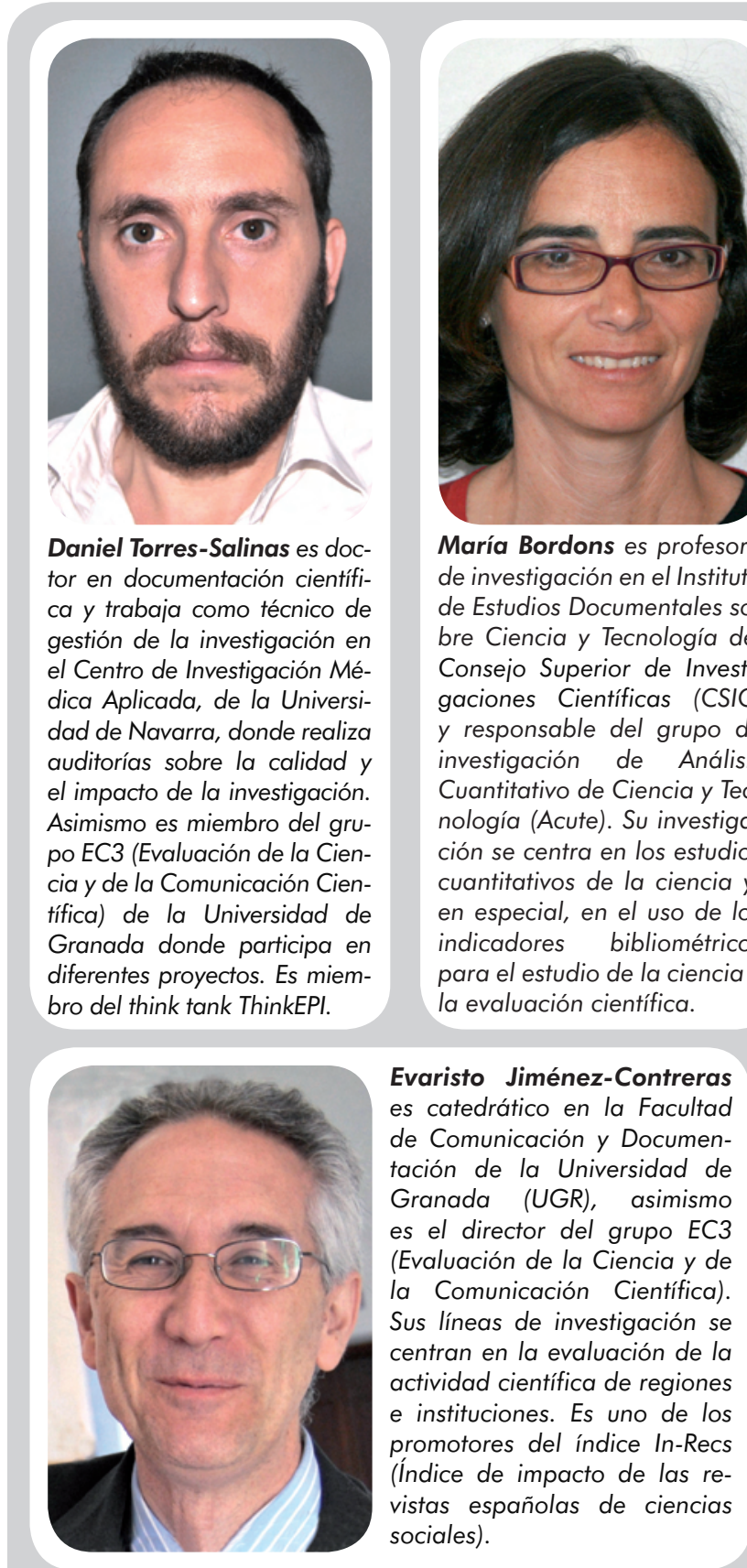

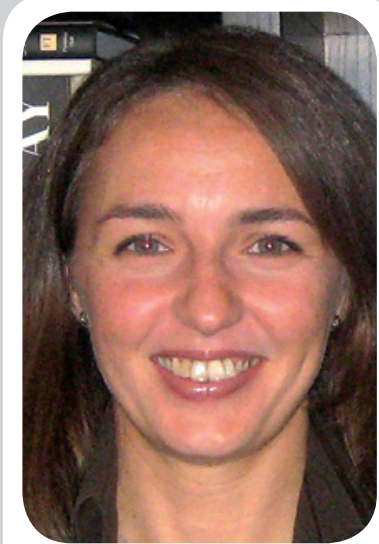

Elea Giménez-Toledo, doctora en documentación por la Univ. Carlos III de Madrid, es científica titular del CSIC y responsable del grupo de investigación de evaluación de publicaciones científicas (EPUC) del Instituto de Estudios Documentales sobre Ciencia y Tecnología (ledcyt). Su principal línea de investigación es la evaluación de publicaciones científicas en ciencias humanas y sociales. Coordina las plataformas RESH, DICE y la parte española de Latindex.

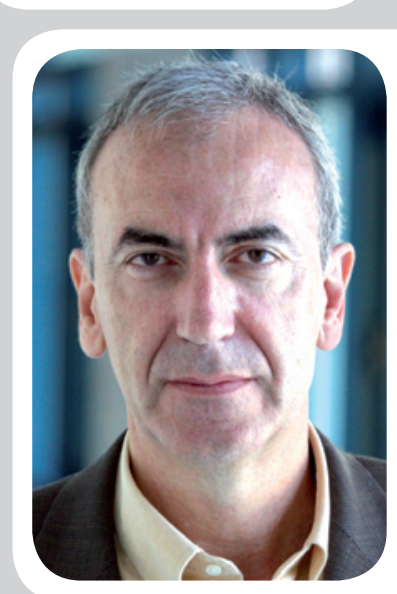

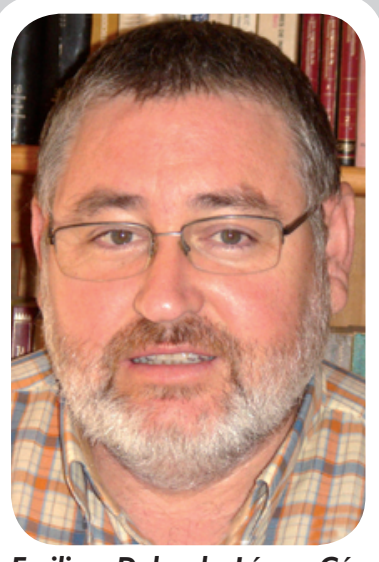

Emilio Delgado-López-Cózar es profesor de metodología de la investigación en la Facultad de Comunicación y Documentación de la Universidad de Granada y miembro del grupo EC3. Sus líneas de investigación se centran en la evaluación de revistas científicas y de la ciencia, el estudio de la investigación en ByD, y la evaluación del rendimiento científico. Es uno de los promotores del índice In-Recs (Índice de impacto de las revistas españolas de ciencias sociales).

Elías Sanz-Casado es catedrático de biblioteconomía y documentación de la Universidad Carlos III de Madrid (UC3M) y director del LEMI (Laboratorio de Estudios Métricos de Información). Sus líneas de investigación se centran en los estudios bibliométricos, cienciométricos $y$, concretamente en la elaboración de indicadores para la evaluación científica de áreas de conocimiento e instituciones.

http://lemi.uc3m.es/content/ dr-elias-sanz-casado

Resumen: Se presenta una propuesta de clasificación cualitativa de revistas científicas especializadas en ciencias sociales y humanas. El principal objetivo de la Clasificación integrada de revistas científicas (CIRC) es ser una herramienta para obtener indicadores bibliométricos sobre la calidad de las publicaciones para distintos niveles de agregación. Para su elaboración se ha contado con la participación y el consenso de distintos expertos en bibliometría. La clasificación resultante consta de cuatro grupos jerárquicos $(g A, g B, g C, g D)$ en función de la visibilidad de las revistas y un quinto 
grupo de excelencia (gEx). Para crear los grupos y determinar la calidad de las revistas se han utilizado listados, bases de datos y rankings referenciados por Aneca y Cneai en sus normativas. Finalmente se ilustra el uso de CIRC aplicándola a la producción científica en revistas de los departamentos de ciencias sociales y humanas de la Universidad de Granada.

Palabras clave: Revistas científicas, Indicadores bibliométricos, Ciencias sociales, Ciencias humanas, Clasificaciones de revistas, Rankings, Aneca, Cneai, Evaluación científica.

Title: Integrated scientific journals classification (CIRC): A proposal for categorization of journals in social sciences and humanities

Abstract: A proposal for a qualitative classification of scientific journals specialized in social sciences and humanities is presented. The main objective of CIRC (in Spanish, "Clasificación integrada de revistas cientificas") is to become a tool to obtain bibliometrics indicators for different levels of aggregation based on the quality of publications. The ranking is the result of consensus of experts in the field of bibliometrics and research evaluation. The resulting classification consists of four groups of journals $(g A, g B, g C, g D)$ configured according to the visibility of the journals; a fifth group of excellence was created with the most relevant journals $(g E x)$. To create groups and determine quality of the journals, various lists and databases referenced in Cneai and Aneca standards were used. Finally, we illustrate the use of CIRC applying it to the scientific production of the departments of Social Sciences at the University of Granada.

Keywords: Scientific journals, Bibliometric indicators, Social sciences, Human sciences, Journal classifications, Rankings, Aneca, Cneai, Research evaluation.

Torres-Salinas, Daniel; Bordons, María; Giménez-Toledo, Elea; Delgado-López-Cózar, Emilio; Jiménez-Contreras, Evaristo; Sanz-Casado, Elías. “Clasificación integrada de revistas científicas (CIRC): propuesta de categorización de las revistas de ciencias sociales y humanas". El profesional de la información, 2010, noviembre-diciembre, v. 19, n. 6, pp. 675-683.

DOI: 10.3145/epi.2010.nov.15

\section{Introducción}

LA OBTENCIÓN DE INDICADORES bibliométricos a distintos niveles de agregación para la evaluación de áreas científicas como las ciencias exactas, experimentales, biomedicina y, en menor medida, las ingenierías es un problema casi resuelto, al menos, en lo que respecta a los indicadores más básicos y las fuentes de información más adecuadas (Moed, 2005).

Hay consenso en la utilización de las diferentes bases de datos que integran la plataforma $\mathrm{Web}$ of knowledge (WoK) como herramientas adecuadas para establecer el volumen productivo, la citación y el impacto de las revistas de cualquier agente científico. Sin embargo este consenso sobre la utilidad de los productos de Thomson Reuters se reduce considerablemente si se desea valorar la producción científica en revistas de las áreas de ciencias sociales y especialmente de las ciencias humanas. A ello contribuyen distintos factores ampliamen- te recogidos en la literatura (Hemlin; Gustafsson, 1996; Glänzel; Schoepflin, 1999; Zitt et al., 2003; Hicks, 2004; Archambault et al., 2006; Nederhof, 2006; Huang; Chang, 2008, Iribarren-Maestro et al., 2009), entre los que se puede señalar que las revistas nacionales, especialmente relevantes en estas áreas por la mayor orientación nacional de la investigación en las ciencias sociales, y sobre todo en las humanas, tienen una peor cobertura en estas bases de datos. Esta situación genera un enorme desequilibrio ya que mientras se pueden obtener retratos bastantes acertados de la actividad científica en ciencias naturales, los indicadores bibliométricos y las fuentes de información habituales pierden parte de su eficacia en las ciencias sociales y humanas. En consecuencia, la información que aportan los estudios bibliométricos tradicionales es menor en estas áreas, en las que resulta más difícil a los gestores de la política científica evaluar la actividad investigadora.
Debido a estas circunstancias en los últimos años ha surgido una serie de productos documentales (directorios, base de datos, clasificaciones), tanto a nivel internacional como nacional, dedicados a determinar la calidad de las revistas científicas para solventar la falta de herramientas e indicadores más allá de los Journal citation reports (Aleixandre-Benavent; Valderrama-Zurián; González-Alcaide, 2007; Giménez-Toledo; Román-Román; Alcaín-Partearroyo, 2007; Rosado-Millán et al., 2007). Al mismo tiempo Elsevier ha lanzado al mercado Scopus, un índice de citas y base de datos multidisciplinar con mayor cobertura que aquellas que integran la Web of science (WoS) (Gavel; Iselid, 2008). En líneas generales los distintos productos se han elaborado con la intención de esclarecer y analizar la situación de las revistas de ciencias sociales y humanas en determinadas especialidades científicas y de dominios geográficos concretos. Otra característica es que muchos de estos productos utilizan indicadores y 
perspectivas de análisis de diferente naturaleza, bien empleando medidas cercanas al Impact factor (DelgadoLópez-Cózar et al., 2005; TorresSalinas; Jiménez-Contreras, 2010; Delgado-López-Cózar; Ruiz-Pérez; Jiménez-Contreras, 2010), analizando el grado de normalización, funcionamiento y composición editorial de las revistas (GiménezToledo et al., 2009; Cetto, 1998; Alcaín-Partearroyo; Román-Román; Giménez-Toledo, 2008), o incluso clasificando las revistas en función del juicio cualitativo de expertos a partir de una serie de parámetros (ESF Humanities Unit, 2009). Sin embargo hasta el momento no es posible utilizar ninguna de estas herramientas de forma global, por lo que su aprovechamiento desde un punto de vista estrictamente bibliométrico sólo permite obtener retratos parciales si lo aplicamos por ejemplo a la evaluación de universidades o departamentos (Torres-Salinas; DelgadoLópez-Cózar; Jiménez-Contreras, 2009). Aunque estas plataformas aún no han sido aprovechadas por la comunidad bibliométrica para elaborar indicadores similares a los utilizados en ciencias naturales, sí que han sido útiles ya que gran parte de las mismas se han transformado en instrumentos de referencia de diferentes agencias de evaluación.

En el caso español las agencias encargadas de evaluar la producción científica del personal investigador de universidades y opis (organismos públicos de investigación) han sido las más activas a la hora de incorporar entre sus criterios las diferentes clasificaciones y rankings de revistas, sobre todo en lo que respecta a las ciencias sociales y las humanas. Tanto Aneca como Cneai se refieren en sus normativas y principios de evaluación a varios de estos productos. Así Ane$c a$, para las ramas de ciencias sociales/jurídicas y arte/humanidades señala -aparte de los productos de Thomson Reuters- DICE (Difusión y calidad editorial de las revistas españolas de humanidades y ciencias sociales y jurídicas), plataforma financiada por la propia Aneca, y Latindex (Aneca, 2008). Por otro lado Cneai en sus últimos criterios publicados en su Resolución de 18 de nov. de 2009 (BOE, 2009) para los campos 7, 8, 9, 10 y 11 especifica que podrán considerarse también los artículos publicados en revistas listadas en otras bases de datos nacionales o internacionales haciendo mención explícita a las siguientes: ERIH, In-Recs, Latindex, Scopus y DICE-Cindoc. Los criterios de las agencias son por tanto un punto de partida necesario a la hora de detectar cuáles son las herramientas de evaluación que más consenso generan entre la comunidad científica y aquellas que sin duda deberían considerarse en las construcción de indicadores bibliométricos al ser esenciales en la política científica española.

Por ello teniendo en cuenta la dificultad para obtener indicadores bibliométricos en ciencias sociales y humanas y aprovechando los recursos existentes, en este trabajo se propone como objetivo la construcción de una clasificación de revistas científicas en función de su calidad integrando los productos de evaluación existentes considerados positivamente por las diferentes agencias de evaluación nacionales.

Se pretende asimismo que el modelo sea operativo, es decir que se construya una base de datos con la clasificación resultante y que ésta pueda ser integrada en otras aplicaciones para finalmente facilitar la construcción de indicadores bibliométricos destinados a la evaluación y descripción de los resultados de investigación de diversos agentes científicos como universidades, departamentos o investigadores.

La clasificación que se propone nace con el fin de generar un instrumento de medida común que pueda ser empleado por los diferentes grupos de investigación sobre biblio- metría y evaluación de la ciencia que existen actualmente en España de forma que se emplee una clasificación estandarizada que permita realizar comparaciones y compartir información. Por ello, esta propuesta es resultado de un grupo de trabajo formado por diferentes expertos nacionales en el ámbito de la bibliometría pertenecientes a la Universidad de Navarra, el Instituto de Estudios Documentales sobre Ciencia y Tecnología (Iedcyt-CSIC), la Universidad Carlos III de Madrid y la Universidad de Granada.

\section{"Los indicadores \\ bibliométricos y las \\ fuentes de información habituales son \\ inadecuados para evaluar \\ las ciencias sociales y humanas"}

\section{Selección de productos y definición de los grupos}

Dentro de la amplia oferta de productos de evaluación de revistas que existen en la actualidad en primer lugar se seleccionaron todos los empleados por Cneai y Aneca. El listado completo de los mismos, que incluye 12 productos, se muestra en la tabla 1. Todos, a excepción del Scimago journal rank (SJR) y el Índice de impacto de las revistas españolas de ciencias jurídicas (InRecj) son mencionados explícitamente por las agencias. El primero ha sido incorporado porque ordena las revistas indexadas en Scopus en función del SJR y ofrece un complemento a los Impact factors del $J C R$ para algunas áreas de ciencias humanas, y el segundo porque está elaborado con la misma metodología que In-Recs pero exclusivamente dedicado al derecho, que es una de las áreas especialmente necesitadas de marcos de referencia. Dentro 
del corpus de productos establecido encontramos rankings de revistas en función del número de citas como el Journal citation reports (JCR) o el Índice de impacto de las revistas españolas de ciencias sociales (In$R e c s)$, directorios que nos ofrecen información cualitativa como el Catálogo Latindex o las master lists de las revistas que indexan bases de datos como Social science citation index ya que debido al exigente proceso de selección la inclusión en este tipo de bases se considera de por sí un criterio de calidad (RuizPérez et al., 2006).

Otra de las características básicas del conjunto de herramientas de la tabla 1 es que se cubren revistas de distintas nacionalidades y regiones geográficas. Existen productos con las revistas más significativas a nivel mundial, normalmente anglosajonas, como ocurre con las master lists del Arts \& humanities citation index $(A \& H C I)$ y Social sciences citation index (SSCI) pero también quedan representadas las revistas europeas, sobre todo en el ámbito de las humanidades, con el European reference index for the humanities $(E R I H)$. Las revistas españolas quedan ampliamente cubiertas, no sólo por aquellas que ya están presentes en los productos internacionales, sino por la inclusión de In-Recs, In-Recj y DICE dedicados exclusivamente a revistas nacionales. Por último el ámbito iberoamericano, y sobre todo Latinoamérica, también queda incluido gracias a Latindex especializado exclusivamente en este dominio geográfico.

Una vez seleccionados los productos con los cuales se iba a trabajar se estableció a nivel teórico una clasificación jerárquica donde se incluyeran las revistas. En concreto se buscaba una clasificación sencilla, capaz de generar indicadores fácilmente interpretables y apta para ser implementada sin dificultad en diferentes sistemas de información para la obtención de in- dicadores bibliométricos y, lo más importante, que permitiese integrar en un solo esquema de categorización los distintos rankings y listados existentes. Para ello se decidió establecer cuatro grupos siguiendo así el modelo de las ciencias que con frecuencia utiliza los cuartiles del JCR o el de clasificaciones anteriores realizadas a nivel nacional (ANEP/Fecyt, 2008). Asimismo la clasificación trataría de ser una herramienta que pudiera representar las estrategias de publicación de los agentes a evaluar; así se describen en la literatura al menos tres estrategias diferentes:

- una en la que se busca publicar en revistas de la base de datos de la especialidad;

- una segunda donde se publica más selectivamente utilizando criterios como la citación; y

- una tercera cuyo objetivo es alcanzar las publicaciones de mayor prestigio definido éste a través de indicadores como el Impact factor (Bordons, 2004).

Teniendo en cuenta todos estos aspectos, los principios rectores de $C I R C$ han sido la citación que obtienen las revistas, y su cumplimiento de los estándares de calidad editorial. Con ello, la definición teórica de los grupos ha sido la siguiente:

- Grupo A (gA): integrado por las revistas científicas de mayor nivel. Pertenecerían al mismo las revistas internacionales de mayor prestigio que han superado procesos de evaluación muy exigentes para el ingreso en diferentes bases de datos.

- Grupo B (gB): compuesto por revistas científicas españolas de calidad pero que no alcanzan un alto nivel de internacionalización aunque son revistas que reciben cierto grado de citación y que respetan los estándares de publicación. Asimismo forman parte de este grupo aquellas revistas científicas internacionales con un menor pero aceptable grado de prestigio y difusión.

- Grupo C ( $\mathrm{gC}$ ): se incluirían en este grupo las revistas científicas españolas de segundo orden que, o bien son poco citadas, o bien no cumplen con los estándares de publicación científica. También se incluyen las revistas internacionales de menor relevancia.

- Grupo D $(\mathrm{gD})$ : este último grupo estaría conformado por todas aquellas publicaciones no incluidas en ninguna de las categorías anteriores $\mathrm{y}$, por tanto, con un dudoso status científico.

Se consideró un quinto grupo de revistas independiente y complementario de los anteriores, destinado a identificar las mejores publicaciones:

- Grupo de excelencia (gEx): integrado por las revistas con mayor grado de impacto científico, entendiendo como tales las posicionadas en el primer cuartil de los rankings internacionales de citación.

\section{"En España Cneai y Aneca han sido activas incorporando en sus criterios de evaluación las diferentes clasificaciones y rankings de revistas"}

\section{Asignación de revistas a la clasificación}

A continuación se procedió a la asignación de las revistas indexadas en los diferentes productos a los cuatro grupos definidos $(\mathrm{gA}, \mathrm{gB}$, $\mathrm{gC}, \mathrm{gD}$, ) y al grupo de excelencia (gEx) para lo cual los diferentes miembros del equipo se convocaron en diferentes sesiones de trabajo y discusión durante el año 2010. A continuación se muestra como han quedado los grupos: 


\begin{tabular}{|c|c|c|c|c|c|c|c|c|c|}
\hline $\begin{array}{c}\text { Producto de } \\
\text { evaluación de } \\
\text { revistas científicas }\end{array}$ & $\begin{array}{c}\text { Tipo de } \\
\text { producto } \\
\text { documental }\end{array}$ & $\begin{array}{l}\text { Acróni- } \\
\text { mo }\end{array}$ & $\begin{array}{c}\text { Cobertura } \\
\text { geográfica }\end{array}$ & $\begin{array}{l}\text { Especialización } \\
\text { temática }\end{array}$ & Productor & $\begin{array}{l}\text { Agencias } \\
\text { que lo } \\
\text { reseñan }\end{array}$ & $\begin{array}{l}\text { Principal } \\
\text { indicador }\end{array}$ & $\begin{array}{l}\text { Última } \\
\text { actuali- } \\
\text { zación }\end{array}$ & $\begin{array}{c}\text { Número } \\
\text { de } \\
\text { revistas }\end{array}$ \\
\hline $\begin{array}{l}\text { J ournal citation reports. } \\
\text { Science edition }\end{array}$ & $\begin{array}{l}\text { Ranking de } \\
\text { revistas }\end{array}$ & $J C R$ & Mundial & Ciencias & $\begin{array}{l}\text { Thomson } \\
\text { Reuters }\end{array}$ & $\begin{array}{l}\text { Cneai } \\
\text { Aneca }\end{array}$ & $\begin{array}{l}\text { Impact } \\
\text { factor }\end{array}$ & 2009 & $\begin{array}{r}2.093 \\
\text { (ed. } 2009)\end{array}$ \\
\hline $\begin{array}{l}\text { Journal citation reports. } \\
\text { Social sciences edition }\end{array}$ & $\begin{array}{l}\text { Ranking de } \\
\text { revistas }\end{array}$ & $J C R$ & Mundial & Ciencias sociales & $\begin{array}{l}\text { Thomson } \\
\text { Reuters }\end{array}$ & $\begin{array}{l}\text { Cneai } \\
\text { Aneca }\end{array}$ & $\begin{array}{l}\text { Impact } \\
\text { factor }\end{array}$ & 2009 & $\begin{array}{r}604 \\
\text { (ed. 2009) }\end{array}$ \\
\hline $\begin{array}{l}\text { Art \& humanities } \\
\text { citation index. Source } \\
\text { publication list }\end{array}$ & Master list & $\mathrm{A} \& \mathrm{HCl}$ & Mundial & Ciencias humanas & $\begin{array}{l}\text { Thomson } \\
\text { Reuters }\end{array}$ & $\begin{array}{l}\text { Cneai } \\
\text { Aneca }\end{array}$ & -- & 2010 & 1.528 \\
\hline $\begin{array}{l}\text { Science citation index. } \\
\text { Source publication list }\end{array}$ & Master list & $\mathrm{SCl}$ & Mundial & Ciencias & $\begin{array}{l}\text { Thomson } \\
\text { Reuters }\end{array}$ & $\begin{array}{l}\text { Cneai } \\
\text { Aneca }\end{array}$ & -- & 2010 & 8.025 \\
\hline $\begin{array}{l}\text { Social sciences } \\
\text { citation index. Source } \\
\text { publication list }\end{array}$ & Master list & $\mathrm{SSCl}$ & Mundial & Ciencias sociales & $\begin{array}{l}\text { Thomson } \\
\text { Reuters }\end{array}$ & $\begin{array}{l}\text { Cneai } \\
\text { Aneca }\end{array}$ & -- & 2010 & 2.643 \\
\hline $\begin{array}{l}\text { Índice de impacto de las } \\
\text { revistas españolas de } \\
\text { ciencias sociales }\end{array}$ & $\begin{array}{l}\text { Ranking de } \\
\text { revistas }\end{array}$ & In-Recs & España & Ciencias sociales & \begin{tabular}{|l|} 
Grupo EC3. \\
Universidad \\
de Granada
\end{tabular} & Cneai & $\begin{array}{l}\text { Índice de } \\
\text { impacto }\end{array}$ & 2008 & 611 \\
\hline $\begin{array}{l}\text { Índice de impacto de las } \\
\text { revistas españolas de } \\
\text { ciencias jurídicas }\end{array}$ & $\begin{array}{l}\text { Ranking de } \\
\text { revistas }\end{array}$ & In-Recj & España & Ciencias jurídicas & $\begin{array}{l}\text { Grupo EC3. } \\
\text { Universidad } \\
\text { de Granada }\end{array}$ & - & $\begin{array}{l}\text { Índice de } \\
\text { impacto }\end{array}$ & 2008 & 255 \\
\hline $\begin{array}{l}\text { Difusión de las revistas } \\
\text { españolas de ciencias } \\
\text { sociales y humanas }\end{array}$ & $\begin{array}{l}\text { Directorio } \\
\text { cualitativo }\end{array}$ & DICE & España & $\begin{array}{l}\text { Ciencias sociales } \\
\text { Ciencias humanas } \\
\text { Ciencias jurídicas }\end{array}$ & ledcyt & $\begin{array}{l}\text { Cneai } \\
\text { Aneca }\end{array}$ & $\begin{array}{l}5 \text { criterios } \\
\text { calidad }\end{array}$ & continua & 1.832 \\
\hline $\begin{array}{l}\text { European reference } \\
\text { index for the humanities }\end{array}$ & $\begin{array}{l}\text { Clasificación } \\
\text { cualitativa }\end{array}$ & ERIH & Mundial & Ciencias humanas & $\begin{array}{l}\text { European } \\
\text { Science } \\
\text { Foundation }\end{array}$ & Cneai & $\begin{array}{l}\text { Clasificación } \\
\text { ABC }\end{array}$ & 2007 & 5.119 \\
\hline Catálogo Latindex & $\begin{array}{l}\text { Directorio } \\
\text { cualitativo }\end{array}$ & Latindex & $\begin{array}{l}\text { Iberoaméri- } \\
\text { ca }\end{array}$ & $\begin{array}{l}\text { Ciencias sociales } \\
\text { Ciencias humanas } \\
\text { Ciencias }\end{array}$ & $\begin{array}{l}\text { Producto } \\
\text { cooperativo }\end{array}$ & $\begin{array}{l}\text { Cneai } \\
\text { Aneca }\end{array}$ & $\begin{array}{l}33 \text { criterios } \\
\text { de calidad }\end{array}$ & continua & 3.814 \\
\hline Scopus. List of titles & Master list & Scopus & Mundial & $\begin{array}{l}\text { Ciencias sociales } \\
\text { Ciencias humanas } \\
\text { Ciencias }\end{array}$ & Elsevier & Cneai & -- & 2010 & 17.160 \\
\hline $\begin{array}{l}\text { Scimago journal rank } \\
\text { (sólo arts \& humanities) }\end{array}$ & $\begin{array}{l}\text { Ranking de } \\
\text { revistas }\end{array}$ & SJ R & Mundial & Ciencias humanas & Elsevier & -- & SJ R & 2008 & $\begin{array}{r}1.291 \\
\text { (ed. 2008) }\end{array}$ \\
\hline
\end{tabular}

Tabla 1. Características básicas de los productos sobre revistas científicas seleccionados para la construcción de la clasificación

\section{Grupo A}

- Indexadas en Science citation index, Social sciences citation index o Arts \& humanities citation index según los master lists de 2010.

- Indexadas en las listas European reference index for the humanities (European Science Foundation) con un calificación de A.

\section{Grupo B}

- Indexadas en el primer cuartil según promedio de citas de cualquiera de las categorías del Índice de impacto de las revistas españolas de ciencias sociales o del Índice de impacto de las revistas españolas de ciencias jurídicas (Grupo EC3). Se toma como referencia los impactos acumulativos de los años 2004-2008.

- Indexadas en DICE (Difusión de las revistas españolas de cien-

\section{" $\mathrm{CIRC}$ es un instrumento de medida común que puede ser empleado por los diferentes grupos de investigación sobre bibliometría"}

cias sociales y humanas) (Iedcyt) y que cumplen con el requisito de contar con evaluación por expertos y además estar presentes en el $\mathrm{Ca}$ tálogo Latindex.

- Indexadas en la base de datos Scopus según su List of titles de abril de 2010 y catalogadas en las categorías Social sciences (code 3300) y Arts and humanities (code 1200).

- Indexadas en las listas European reference index for the huma- nities (ESF) con un calificación de $\mathrm{B}$ o $\mathrm{C}$.

\section{Grupo C}

- Indexadas en el segundo, tercer o cuarto cuartil según promedio de citas de cualquiera de las categorías del Índice de impacto de las revistas españolas de ciencias sociales o del Índice de impacto de las revistas españolas de ciencias jurídicas (Grupo EC3). Se toma como referencia los impactos acumulativos de los años 20042008.

- Indexadas en DICE (Difusión de las revistas españolas de ciencias sociales y humanas) (Ie$d c y t$ ) pero sin cumplir con el requisito de contar con evaluación por expertos.

- Indexadas en el Catálogo Latindex 


\section{Grupo D}

- Cualquier revista que no está indexada en alguno de los productos reseñados anteriormente.

Para la asignación de las revistas a los diferentes grupos siempre se ha favorecido la mejor clasificación obtenida por las mismas. Por ejemplo, El profesional de la información, que está indexada en SSCI (gA) y en Scopus (gB), teniendo en cuenta el criterio anterior dicha revista queda clasificada en gA. Finalmente junto a estos grupos se creó un quinto nivel independiente de los anteriores (aunque subconjunto de gA) destinado a identificar las revistas muy relevantes en las áreas de ciencias sociales y humanas:

\section{Grupo excelencia}

- Para ciencias sociales: revistas indexadas en el primer cuartil según el Impact factor de cualquiera de las categorías del Journal citation reports (Thomson Reuters).

- Para ciencias humanas: revistas indexadas en el Scimago journal rank (SJR, Elsevier) en las áreas arts \& humanities y que están al mismo tiempo o bien en el $A \& H C I$ o bien en ERIH clasificadas como A.

En última instancia los productos seleccionados fueron descargados en sus últimas versiones disponibles durante julio de 2010, creándose una base de datos con los mismos depurada de errores. En la tabla 2 se presenta el número de revistas que conforman los diferentes grupos de la que finalmente se ha denominado Clasificación integrada de revistas científicas (CIRC) y sus productos de procedencia. El número de revistas procesadas y asignadas a las diferentes agrupaciones ha sido de 19.906, si se consideran todas las revistas; y de 12.318 si se prescinde de las revistas de ciencias incluidas en $S C I$ y JCR (usadas sólo ocasionalmente por los investigadores de ciencias sociales y humanas) y sólo se consideran las revistas especializadas en ciencias sociales y humanidades. El 34\% de las 12.318 revistas están catalogadas como gA, el $35 \%$ como gB y el $30 \%$ como gC. El grupo de excelencia lo componen, si se excluyen las revistas de ciencias, 883 revistas, es decir un $7 \%$ del total de revistas analizadas.

\section{Ejemplo de la utilización de la clasificación}

Con el objetivo de probar la utilidad de esta clasificación para la generación de indicadores bibliométricos se tomaron los trabajos publicados en revistas de los 30 departamentos de Ciencias Sociales y Humanas de la Universidad de Granada con un mayor número de contribuciones. El período cronológico se limitó a los años 2005-2009. La información sobre las publicaciones de dichos departamentos proviene del Sistema de Información Científica de Andalucía.

En los gráficos 1 y 2 se ofrecen los resultados finales. En el primero se muestra el número bruto de trabajos según la clasificación $C I R C$ así como aquellos indexados en revistas de excelencia. En el segundo se muestra el porcentaje de documentos en cada una de las categorías. Tanto en uno como en otro se puede identificar claramente cuál es el perfil o estrategia de publicación de cada uno de los departamentos. Así se distinguen aquellos con una clara vocación internacional $(\mathrm{gA})$ y de excelencia (gEx) de los que publican en revistas de menor entidad ( $\mathrm{gB}$ y gC) e incluso de aquellos que lo hacen en revistas científicas de menor rango o publicaciones no científicas $(\mathrm{gD})$.

\section{"Es una clasificación sencilla que integra distintos rankings y listados, capaz de generar indicadores fácilmente interpretables"}

\begin{tabular}{|c|c|c|c|c|c|c|}
\hline & gA & gB & gC & gD & Total & gEx** \\
\hline Journal citation reports. Science edition* & No aplica & No aplica & No aplica & No aplica & & 2.093 \\
\hline J ournal citation reports. Social sciences edition** & No aplica & No aplica & No aplica & No aplica & & 604 \\
\hline Art \& humanities citation index. Source publication list & 1.527 & No aplica & No aplica & No aplica & & No aplica \\
\hline Science citation index. Source publication list & 8.026 & No aplica & No aplica & No aplica & & No aplica \\
\hline Social sciences citation index. Source publication list & 2.643 & No aplica & No aplica & No aplica & & No aplica \\
\hline Índice de impacto de las revistas españolas de ciencias sociales & No aplica & 120 & 497 & No aplica & & No aplica \\
\hline Índice de impacto de las revistas españolas de ciencias jurídicas & No aplica & 58 & 201 & No aplica & & No aplica \\
\hline Difusión de las revistas españolas de ciencias sociales y humanas & No aplica & 651 & 1.181 & No aplica & & No aplica \\
\hline European reference index for the humanities & 1.112 & 4.224 & No aplica & No aplica & & No aplica \\
\hline Catálogo Latindex & No aplica & No aplica & 3.814 & No aplica & & No aplica \\
\hline Scopus. List of titles. Social sciences y arts $\&$ humanities & No aplica & 2.673 & No aplica & No aplica & & No aplica \\
\hline $\begin{array}{l}\text { (ERIH - tipo A) o }(\mathrm{A} \& \mathrm{HCl}) \text { y SCImago journal rank. } \\
\text { Arts \& humanities }\end{array}$ & No aplica & No aplica & No aplica & No aplica & & 291 \\
\hline № revistas sin duplicados o preasignados otros grupos & 11.816 & 4.306 & 3.784 & $\begin{array}{l}\text { Resto de } \\
\text { revistas }\end{array}$ & 19.906 & 2.858 \\
\hline № revistas sin duplicados excluyendo $\mathrm{SCl}$ y JCR science & 4.228 & 4.306 & 3.784 & $\begin{array}{l}\text { Resto de } \\
\text { revistas }\end{array}$ & 12.318 & 883 \\
\hline
\end{tabular}

Tabla 2. Número de revistas según los grupos de la clasificación CIRC y los productos de evaluación de revistas de referencia 


\begin{tabular}{|c|c|c|c|c|c|c|c|}
\hline & Departamento & № gA & №gB & №gC & № gD & № gEX & \%gEX \\
\hline \multirow{15}{*}{$\frac{n}{\frac{n}{2}}$} & HISTORIA DEL ARTE Y MUSICA & 9 & 64 & 20 & 62 & 0 & $0 \%$ \\
\hline & LITERATURA ESPAÑOLA & 25 & 12 & 27 & 90 & 3 & $2 \%$ \\
\hline & FILOLOGÍAS INGLESA Y ALEMANA & 14 & 35 & 31 & 61 & 1 & $1 \%$ \\
\hline & FILOLOGÍA GRIEGA Y FILOLOGÍA ESLAVA & 6 & 21 & 33 & 55 & 0 & $0 \%$ \\
\hline & ESTUdIOS SEMÍTICOS & 14 & 12 & 44 & 43 & 3 & $3 \%$ \\
\hline & TRADUCCIÓN E INTERPRETACIÓN & 23 & 33 & 5 & 44 & 2 & $2 \%$ \\
\hline & PREHISTORIA Y ARQUEOLOGÍA & 18 & 19 & 30 & 36 & 4 & $4 \%$ \\
\hline & HISTORIA MEDIEVAL & 3 & 14 & 29 & 29 & 1 & $1 \%$ \\
\hline & LINGÜÍSTICA GEN.Y TEORIAA DE LA LITE. & 3 & 10 & 8 & 52 & 0 & $0 \%$ \\
\hline & FILOSOFIA II & 9 & 18 & 14 & 28 & 0 & $0 \%$ \\
\hline & FILOSOFIA I & 29 & 10 & 19 & 12 & 10 & $17 \%$ \\
\hline & FILOLOGÍA FRANCESA & 5 & 24 & 5 & 12 & 3 & $7 \%$ \\
\hline & FILOLOGÍAS: ROM., ITAL., GALLEGO Y CAT. & 4 & 15 & 2 & 22 & 1 & $2 \%$ \\
\hline & FILOLOGÍA LATINA & 5 & & 16 & 11 & 0 & $0 \%$ \\
\hline & HISTORIA CONTEMPORÁNEA & 5 & 10 & 12 & 12 & 0 & $0 \%$ \\
\hline \multirow{15}{*}{ 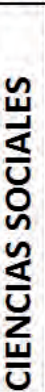 } & PERSONALIDAD, EVALUACIÓN Y TRAT, PSIC. & 221 & 46 & 34 & 48 & 53 & $15 \%$ \\
\hline & DIDÁCTICA Y ORGANIZACIÓN ESCOLAR & 33 & 105 & 63 & 132 & 0 & $0 \%$ \\
\hline & EDUCACIÓN FÍSICA Y DEPORTIVA & 100 & 57 & 63 & 103 & 9 & $3 \%$ \\
\hline & PSIC. EXPERIMENTAL, Y FISIOL, COMPORTAMIENTO & 200 & 8 & 13 & 39 & 77 & $30 \%$ \\
\hline & PSICOLOGÍA EVOLUUTIVA Y DE LA EDUCACIÓN & 67 & 65 & 54 & 42 & 12 & $5 \%$ \\
\hline & BIBLIOTECONOMÍA Y DOCUMENTACIÓN & 123 & 11 & 30 & 30 & 31 & $16 \%$ \\
\hline & PSIC. SOCIAL Y METODOLOGÍA DE LAS C.C. & 99 & 24 & 23 & 38 & 13 & $7 \%$ \\
\hline & DIDÁCTICA DE LA MATEMÁTICA & 14 & 39 & 46 & 64 & 0 & $0 \%$ \\
\hline & TEORIA E HISTORIA ECONOMICA & 51 & 31 & 28 & 49 & 5 & $3 \%$ \\
\hline & DIDÁC. EXPRESIÓN MUSICAL PLÁSTICAbCORPORAL & 34 & 39 & 23 & 35 & 3 & $2 \%$ \\
\hline & ANTROPOLOGÍA SOCIAL & 15 & 33 & 18 & 52 & 2 & $2 \%$ \\
\hline & DIDÁCTICA DE LA LENGUA Y LA LITERATURA & 14 & 40 & 14 & 44 & 0 & $0 \%$ \\
\hline & PEDAGOGÍA & 14 & 35 & 22 & 35 & 0 & $0 \%$ \\
\hline & ECONOMÍA APLICADA & 30 & 30 & 13 & 31 & 6 & $6 \%$ \\
\hline & ORGANIZACIÓN DE EMPRESAS & 47 & 26 & 7 & 23 & 8 & $8 \%$ \\
\hline
\end{tabular}

Gráfico 1. Número de trabajos según clasificación CIRC para los departamentos de las áreas de ciencias sociales y humanas de la Universidad de Granada. Quinquenio 2005-2009

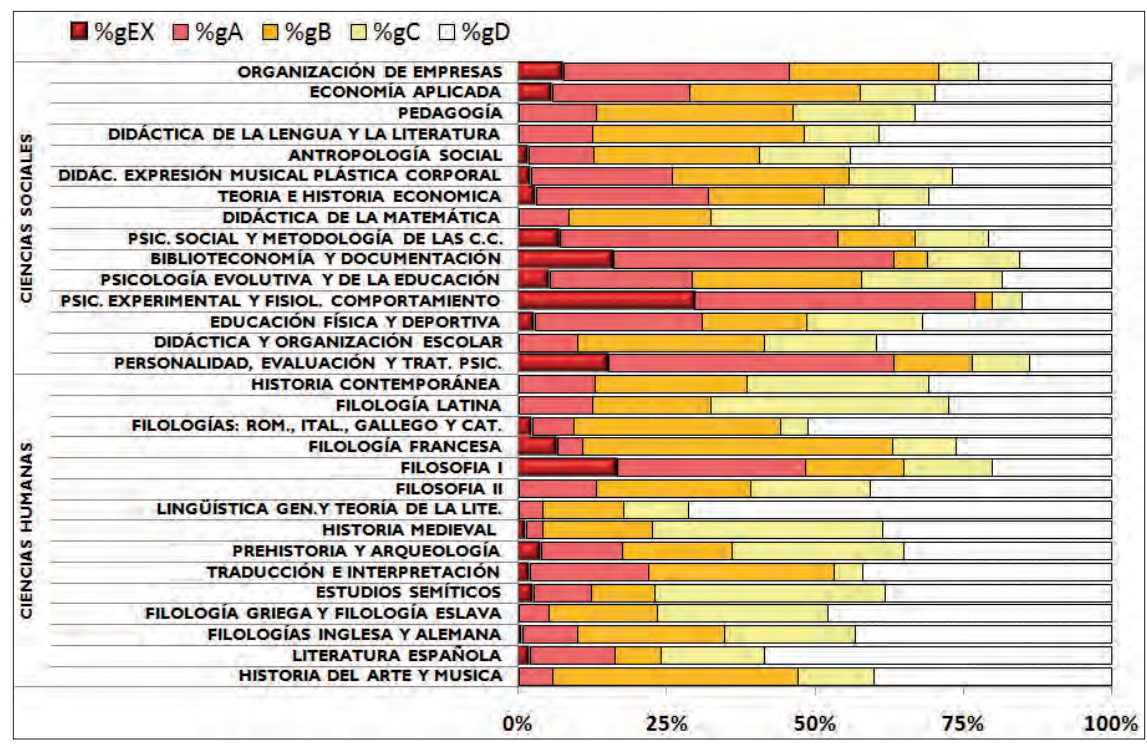

Gráfico 2. Porcentaje de trabajos según clasificación CIRC para los departamentos de las áreas de ciencias sociales y humanas de la Universidad de Granada. Quinquenio 2005-2009

\section{Consideraciones finales}

En este trabajo se ha presentado una propuesta de clasificación de revistas científicas que integra distintos productos sobre revistas (listados, rankings, etc.) existentes actualmente en el mercado de la información científica. Dicha clasificación, que se ha denominado Clasificación de integrada de revistas científicas (CIRC), está orientada a solventar la falta de herramientas para obtener indicadores bibliométricos para el estudio de la de medida estandarizado. Al mismo tiempo la clasificación puede tener un uso práctico en las agencias de evaluación e incluso puede ser incorporada como información complementaria en los diferentes productos que se desarrollan a nivel nacional.

Se prevé que tras esta primera fase el grupo de trabajo realice actualizaciones anuales de la clasificación CIRC de manera que ésta tenga continuidad en el tiempo y pueda ir mejorándose, no sólo incorporando nuevas fuentes que vayan surgiendo, sino también subsanando una serie de limitaciones que se han identificado y que es necesario señalar:

- Se pueden arrastrar las insuficiencias propias de las fuentes que se emplean, como cierto sesgo en las coberturas; aunque la mayor parte de los productos empleados están especializados en ciencias sociales y humanas es necesario la realización de nuevos estudios en especialidades concretas para observar el número total de revistas que las integran así como su distribución en los distintos grupos.

- Se hacen equivaler en peso productos que tienen una naturaleza, finalidad y aplicación distinta por lo que habría que estudiar detenidamente hasta qué punto son equiparables más allá de los propios criterios de las agencias de evaluación. Por ejemplo en la categoría B: impacto en In-Recs y calidad editorial (cumplir criterios Latindex y evaluación por pares en $D I C E$ ).

Por último en este trabajo se ha ilustrado el interés práctico que puede tener una clasificación de este tipo a través de un estudio de la producción científica de los departamentos de Ciencias Sociales y Humanas de la Universidad de Granada, mostrándose la utilidad de la misma para obtener el perfil de publicación de los departamentos y realizar análisis comparativos. 


\section{Referencias}

Aleixandre-Benavent, Rafael; ValderramaZurián, Juan-Carlos; González-Alcaide, Gregorio. "El factor de impacto de las revistas científicas: limitaciones e indicadores alternativos". El profesional de la información, 2007, enero-febrero, v. 16, n. 1, pp. 4-11.

http://www.elprofesionaldelainformacion.com/ contenidos/2007/enero/01.pdf

ANEP/Fecyt. Categorización de revistas científicas españolas de humanidades (ANEP/Fecyt), 2008.

http://humanidades.cchs.csic.es/cchs/epuc/docs/ anep_fecyt.pdf

Alcaín-Partearroyo, María-Dolores; RománRomán, Adelaida; Giménez-Toledo, Elea. "Categorización de las revistas españolas de ciencias sociales y humanas en RESH". Revista española de documentación científica, 2008, v. 31, n. 1, pp. 85-95.

http://redc.revistas.csic.es/index.php/redc/arti cle/view/414/426

Aneca. Programa Academia. Principios y orientaciones para la aplicación de los criterios de evaluación, 2008.

http://www.aneca.es/actividadesevaluacion/eva luacionprofesorado/acreditacion_nacional.aspx

Archambault, Éric; Vignola-Gagne, Étienne; Côté, Grégoire; Larivière, Vincent; Gingras, Ives. "Benchmarking scientific output in the social sciences and humanities: the limits of existing databases". Scientometrics, 2006, v. 68, n. 3, pp. 329-342.

http://www.science-metrix.com/pdf/Benchmar king_SSH.pdf

BOE. Resolución de 18 nov. 2009, de la Presidencia de la Comisión Nacional Evaluadora de la Actividad Investigadora, por la que se establecen los criterios específicos en cada uno de los campos de evaluación, 2009, pp. 102491-102505.

http://www.boe.es/boe/dias/2009/12/01/pdfs/ BOE-A-2009-19218.pdf

Bordons, María. "Hacia el reconocimiento internacional de las publicaciones científicas españolas". Revista española de cardiología, 2004, v. 57, n. 9, pp. 799-802.

http://digital.csic.es/bitstream/10261/11564/1/ 25v57n09a13065646pdf001.pdf

Cetto, Ana-María "Ciencia y producción científica en América Latina. El proyecto Latindex". International microbiology: the official journal of the Spanish Society for Microbiology, 1998, v. 1, n. 3, pp. 181-182.

http://www.im.microbios.org/03 setember98/ $02 \% 20$ Cetto\%20(E).pdf

Delgado-López-Cózar, Emilio; Jiménez-Contreras, Evaristo; Ruiz-Pérez, Rafael; LópezHerrera, Antonio-Gabriel; Gacto-Colorado, María-José; Torres-Salinas, Daniel; MonedaCorrochano, Mercedes; Ruiz-Baños, Rosario; Pérez-Ortega, Juan-Manuel; Bailón-Moreno, Rafael; Poyatos-Huertas, Encarnación; Rodríguez-Gálvez, María-José. "In-Recs: Índice de impacto de las revistas españolas de ciencias sociales. Una nueva herramienta para medir el impacto de la investigación española”. Biblio 3 W, Revista bibliográfica de geografía y ciencias sociales, 2005, marzo, v. 10, n. 574.

http://www.ub.es/geocrit/b3w-574.htm

Delgado-López-Cózar, Emilio; Ruiz-Pérez,
Rafael; Jiménez-Contreras, Evaristo. "Qué es y cómo utilizar el índice de impacto de revistas españolas de ciencias jurídicas". Aula abierta, 2010, v. 38, n. 2, pp. 3-16

http://ec3.ugr.es/publicaciones/INRECJS_Aula_ Abierta.pdf

ESF Humanities Unit. Increasing visibility for a multifaceted humanities research in Europe - the ERIH approach, 2009.

http://www.esf.org/research-areas/humanities/ erih-european-reference-index-for-the-huma nities/erih-initial-lists.html

Gavel, Ylva; Iselid, Lars. "Web of science and Scopus: a journal title overlap study". Online information review, 2008, v. 32, n. 1, pp. 8-21.

Glänzel, Wolfgang; Schoepflin, Urs. "A bibliometric study of reference literature in the sciences and social sciences". Information processing and management, 1999, v. 35, n. 1, pp. 31-44.

Giménez-Toledo, Elea; Román-Román, Adelaida; Alcaín-Partearroyo, María-Dolores "From experimentation to coordination in the evaluation of Spanish scientific journals in the humanities and social sciences". Research evaluation, 2007, v. 16, n. 2, pp. 137-148.

http://www.ingentaconnect.com/content/beech/ rev/2007/00000016/00000002/art00006

Giménez-Toledo, Elea; Román-Román, Adelaida; Perdiguero, Pablo; Palencia, Irene. "The editorial boards of Spanish scholarly journals what are they like? What should they be like?' Journal of scholarly publishing, 2009, v. 40, n. 3 , pp. 287-306.

Hemlin, S.; Gustafsson, M. "Research production in the arts and humanities: a questionnaire study of factors influencing research performance". Scientometrics, 1996, v. 37, n. 3, pp. 417-432.

http://www.springerlink.com/content/lnu 12358 08137316/fulltext.pdf

Hicks, Diana. "The four literatures of social science". En: Moed Henk. Handbook of quantita tive science and technology research. Dordrecht: Kluwer Academic, 2004, pp. 473-496. ISBN 1402027028 .

http://citeseerx.ist.psu.edu/viewdoc/download?d $o i=10 \cdot 1 \cdot 1 \cdot 10.500 \&$ rep $=$ rep $1 \&$ type $=p d f$

Huang, Mu-hsuan; Chang, Yu-wei. "Characteristics of research output in social sciences and humanities from a research evaluation perspec tive". Journal of the American Society for Information Science and Technology, 2008, v. 59, n 11, pp. 1819-1828

Iribarren-Maestro, Isabel; LascurainSánchez, María-Luisa; Sanz-Casado, Elías "The use of bibliometric techniques in evaluating social sciences and humanities". En: Åström, Fredrik; Danell, Rickard; Larsen, Birger; Schneider, Jesper-Wiborg. Celebrating scholarly communication studies: a festschrift for Olle Persson at his 60th birthday. Intl Society for Scientometrics and Informetrics, 2009, pp. 25-37. http://www8.umu.se/inforsk/Bibexcel/olleper sson60.pdf

Moed, Henk F. Citation analysis in research evaluation. Dordrecht: Springer, 2005, ISBN 1402037139.

Nederhof, Anton J. "Bibliometric monitoring of research performance in the social sciences and the humanities: a review". Scientometrics, 2006 , v. 66, n. 1, pp. $81-100$
Rosado-Millán, María-Jesús; Román-Román, Adelaida; Sanz-Casado, Elías; Berges-Torres, Mónica; Gómez-Cedillo, Adolfo; GarcíaGarcía, Francisco; Aguillo, Isidro F. Criterios de clasificación de los medios de difusión de la producción académica y científica universitaria. Madrid: Agencia de Calidad, Acreditación y Prospectiva de las Universidades de Madrid (ACAP), 2008, ISBN 9788461200245.

Ruiz-Pérez, Rafael; Delgado-López-Cózar, Emilio; Jiménez-Contreras, Evaristo. "Criterios del Institute for Scientific Information para la selección de revistas científicas. Su aplicación a las revistas españolas: metodología e indicadores". International journal of clinical and health psychology, 2006, v. 6, n. 2, pp. 401-424. http://redalyc.uaemex.mx/src/inicio/ArtPdfRed. jsp?iCve $=33760211$

Torres-Salinas, Daniel; Jiménez-Contreras, Evaristo. "Introducción y estudio comparativo de los nuevos indicadores de citación sobre revistas científicas en Journal citation reports y Scopus". El profesional de la información, 2010, marzo-abril, v. 19, n. 2, pp. 201-208.

http://elprofesionaldelainformacion.metapress. com

Torres-Salinas, Daniel; Delgado-López-Cózar, Emilio; Jiménez-Contreras, Evaristo. "Análisis de la producción de la Universidad de Navarra en revistas de ciencias sociales y humanidades empleando rankings de revistas españolas y la Web of science". Revista española de documentación cientifica, 2009, v. 32, n. 1, pp. 22-39. http://redc.revistas.csic.es/index.php/redc/ article/download/451/463

Zitt, Michael; Ramanana-Rahary, Suzy; Bassecoulard, Elise. "Correcting glasses help fair comparisons in international science landscape: country indicators as a function of ISI database delineation". Scientometrics, 2003, v. 56, n. 2 , pp. 259-282.

http://www.springerlink.com/content/g40267h 787881601/fulltext.pdf

Daniel Torres-Salinas. Evaluación de la Ciencia y la Comunicación Científica (EC3), Universidad de Granada; y Centro de Investigación Médica Aplicada, Universidad de Navarra.

Avda. Pío XII, 55. 31008 Pamplona, España.

torressalinas@gmail.com

María Bordons. Instituto de Estudios Documentales sobre Ciencia y Tecnología (Iedcyt), Centro de Ciencias Humanas y Sociales (CCHS), Consejo Superior de Investigaciones Científicas (CSIC).

Albasanz, 26-28. 28037 Madrid, España; y Unidad Asociada IedcytLEMI de la Universidad Carlos III de Madrid.

maria.bordons@cchs.csic.es 
Elea Giménez-Toledo. Instituto de Estudios Documentales sobre Ciencia y Tecnología (Iedcyt), Centro de Ciencias Humanas y Sociales (CCHS), Consejo Superior de Investigaciones Científicas.

Albasanz, 26-28. 28037Madrid, Espa$\tilde{n} a ;$ y Unidad Asociada Iedcyt-LEMI de la Univ.Carlos III de Madrid. elea.gimenez@cchs.csic.es
Emilio Delgado-López-Cózar, Evaristo Jiménez-Contreras. Evaluación de la Ciencia y la Comunicación Científica (EC3), Departamento de Biblioteconomía y Documentación, Universidad de Granada.

Campus Cartuja. 18011 Granada, España.

edelgado@ugr.es

evaristo@ugr.es
Elías Sanz-Casado. Unidad Asociada Iedcyt-LEMI de la Universidad Carlos III de Madrid; y Departamento de Biblioteconomía y Documentación, Universidad Carlos III de Madrid,

Calle Madrid, 126. 28903, Getafe (Madrid), España. elias@bib.uc3m.es

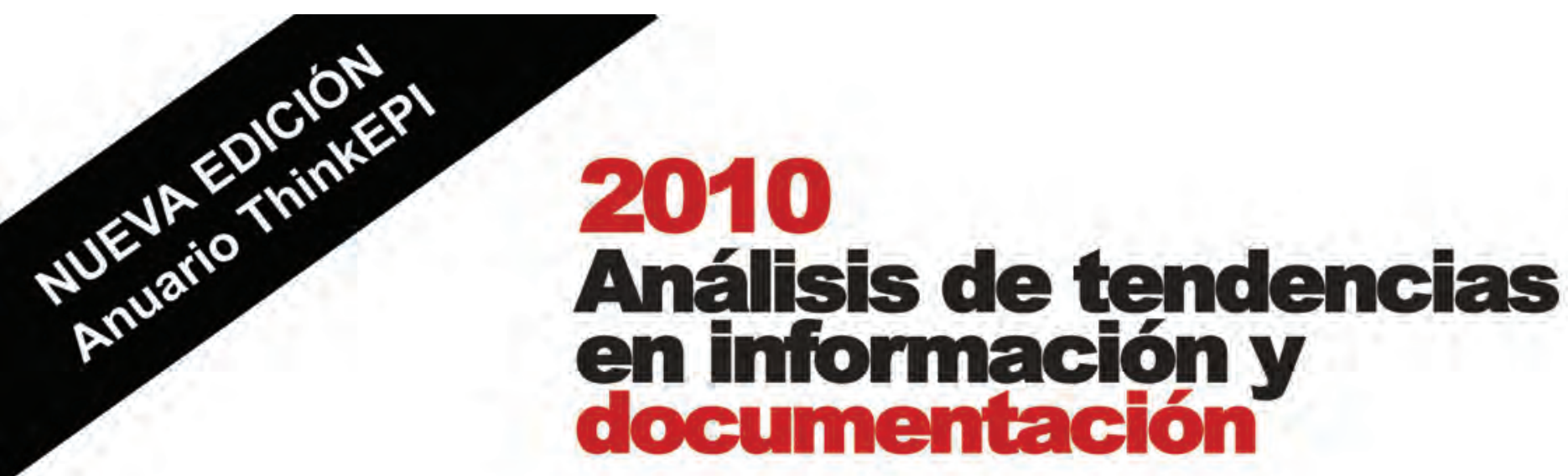

Algunos de los títulos:

Formación universitaria en información y documentación Cristobal Urbano y Sílvia Argudo

Bibliotecas escolares en España José-Antonio Gómez-Hernández

Compartir datos en ciencia: contexto de una oportunidad Daniel Torres-Salinas

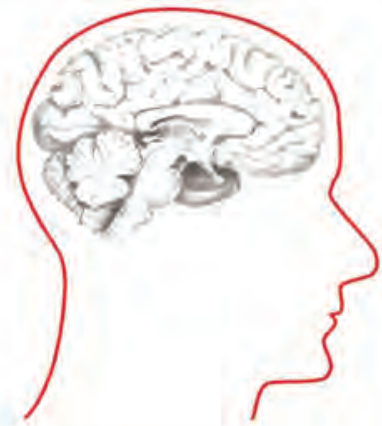

Reflexiones y buenas prácticas en webs académicas personales Isidro F. Aguillo

Software para el acceso a la información. Situación del mercado Ricardo Eíto-Brun

Sobre usabilidad y experiencia de uso Mari-Carmen Marcos

ANUARIO 


\section{MiBjb]joteca}

\section{Actualidad bibliotecaria}

Fotografía momentos de lectura

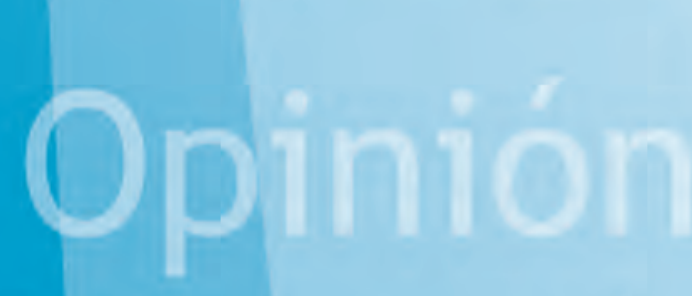

Recomendaciones de lectura Escolares

\section{Rúblicas}

ReDOrta entrevistas Recursos Universitarias

Con pasado

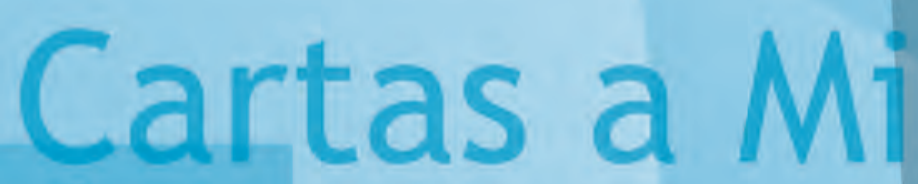

Bibliotecarios

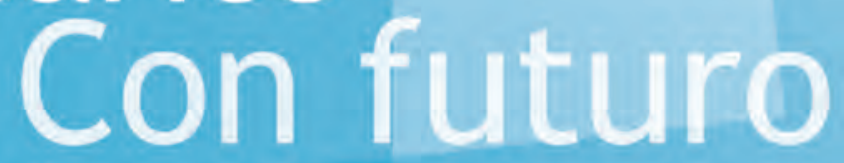

Noticias

Nuevas Tecnologías

Gestión documental

www.mibiblioteca.org 